\title{
What Do Reversible Programs Compute?
}

\author{
Holger Bock Axelsen and Robert Glück \\ DIKU, Department of Computer Science, University of Copenhagen \\ funkstar@diku.dk, glueck@acm.org
}

\begin{abstract}
Reversible computing is the study of computation models that exhibit both forward and backward determinism. Understanding the fundamental properties of such models is not only relevant for reversible programming, but has also been found important in other fields, e.g., bidirectional model transformation, program transformations such as inversion, and general static prediction of program properties.

Historically, work on reversible computing has focussed on reversible simulations of irreversible computations. Here, we take the viewpoint that the property of reversibility itself should be the starting point of a computational theory of reversible computing. We provide a novel semantics-based approach to such a theory, using reversible Turing machines (RTMs) as the underlying computation model.

We show that the RTMs can compute exactly all injective, computable functions. We find that the RTMs are not strictly classically universal, but that they support another notion of universality; we call this RTMuniversality. Thus, even though the RTMs are sub-universal in the classical sense, they are powerful enough as to include a self-interpreter. Lifting this to other computation models, we propose r-Turing completeness as the 'gold standard' for computability in reversible computation models.
\end{abstract}

\section{Introduction}

The computation models that form the basis of programming languages are usually deterministic in one direction (forward), but non-deterministic in the opposite (backward) direction. Most other well-studied programming models exhibit non-determinism in both computation directions. Common to both of these classes is that they are information lossy, because generally a previous computation state cannot be recovered from a current state. This has implications on the analysis and application of these models. Reversible computing is the study of computation models wherein all computations are organized two-way deterministically, without any logical information loss.

Reversible computation models have been studied in widely different areas ranging from cellular automata 11, program transformation concerned with the inversion of programs [19, reversible programming languages 321, the viewupdate problem in bidirectional computing and model transformation [146], static prediction of program properties [15], digital circuit design [18 20], to quantum computing [5]. However, between all these cases, the definition and use of reversibility varies significantly (and subtly), making it difficult to apply

M. Hofmann (Ed.): FOSSACS 2011, LNCS 6604, pp. 42-56 2011.

(C) Springer-Verlag Berlin Heidelberg 2011 
results learned in one area to others. For example, even though reversible Turing machines were introduced several decades ago [4, the authors have found that there has been a blurring of the concepts of reversibility and reversibilization, which makes it difficult to ascertain exactly what is being computed, in later publications.

This paper aims to establish the foundational computability aspects for reversible computation models from a formal semantics viewpoint, using reversible Turing machines (RTMs, 4]) as the underlying computation model, to answer the question: What do reversible programs compute?

In reversible computation models, each atomic computation step must be reversible. This might appear as too restrictive to allow general and useful computations in reversible computation models. On the other hand, it might appear from the seminal papers by Landauer [9] and Bennett 4], that reversibility is not restrictive at all, and that all computations can be performed reversibly. We show that both of these viewpoints are wrong, under the view that the functional (semantical) behavior of a reversible machine should be logically reversible.

This paper brings together many different streams of work in an integrated semantics formalism that makes reversible programs accessible to a precise analysis, as a stepping stone for future work that makes use of reversibility. Thus, this paper is also an attempt to give a precise structure and basis for a foundational computability theory of reversible languages, in the spirit of the semantics approach to computability of Jones 8 .

We give a formal presentation of the reversible Turing machines (Sect. 2), and, using a semantics-based approach (Sect. 3), outline the foundational results of reversible computing (Sect.4). We show the computational robustness of the RTMs under reductions of the number of symbols and tapes (Sect. 5). Following a proof tactic introduced by Bennett, we show that the RTMs can compute exactly all injective, computable functions (Sect. 6). We study the question of universality, and give a novel interpretation of the concept (RTM-universality) that applies to RTMs, and prove constructively that the RTMs are RTM-universal (Sect. 7). We propose $r$-Turing completeness (Sect. 8) as the measure for computability of reversible computation models. Following a discussion of related work (Sect. 9) we present our conclusions (Sect. 10).

\section{Reversible Triple-Format Turing Machines}

The computation model we shall consider here is the Turing machine (TM). Recall that a Turing machine consists of a (doubly-infinite) tape of cells along which a tape head moves in discrete steps, reading and writing on the tape according to an internal state and a fixed transition relation. We shall here adopt a triple format for the rules which is similar to Bennett's quadruple format [4, but has the advantage of being slightly easier to work with 1 .

${ }^{1}$ It is straightforward to translate back and forth between triple, quadruple, and the usual quintuple formats. 
Definition 1 (Turing machine). A TMT is a tuple $\left(Q, \Sigma, \delta, b, q_{s}, q_{f}\right)$ where $Q$ is a finite set of states, $\Sigma$ is a finite set of tape symbols, $b \in \Sigma$ is the blank symbol,

$$
\delta \subseteq(Q \times[(\Sigma \times \Sigma) \cup\{\leftarrow, \downarrow, \rightarrow\}] \times Q)=\Delta
$$

is a partial relation defining the transition relation, $q_{s} \in Q$ is the starting state, and $q_{f} \in Q$ is the final state. There must be no transitions leading out of $q_{f}$ nor into $q_{s}$. Symbols $\leftarrow, \downarrow, \rightarrow$ represent the three shift directions (left, stay, right).

The form of a triple in $\delta$ is either a symbol rule $\left(q,\left(s, s^{\prime}\right), q^{\prime}\right)$ or a shift rule $\left(q, d, q^{\prime}\right)$ where $q, q^{\prime} \in Q, s, s^{\prime} \in \Sigma$, and $d \in\{\leftarrow, \downarrow, \rightarrow\}$. Intuitively, a symbol rule says that in state $q$, if the tape head is reading symbol $s$, write $s^{\prime}$ and change into state $q^{\prime}$. A shift rule says that in state $q$, move the tape head in direction $d$ and change into state $q^{\prime}$. It is easy to see how to extend the definition to $k$-tape machines by letting

$$
\delta \subseteq\left(Q \times\left[(\Sigma \times \Sigma)^{k} \cup\{\leftarrow, \downarrow, \rightarrow\}^{k}\right] \times Q\right) .
$$

Definition 2 (Configuration). The configuration of a TM is a tuple $(q,(l, s, r))$ $\in Q \times\left(\Sigma^{*} \times \Sigma \times \Sigma^{*}\right)=\mathcal{C}$, where $q \in Q$ is the internal state, $l, r \in \Sigma^{*}$ are the parts of the tape to the left and right of the tape head represented as strings, and $s \in \Sigma$ is the symbol being scanned by the tape head2.

Definition 3 (Computation step). A TMT $=\left(Q, \Sigma, \delta, b, q_{s}, q_{f}\right)$ in configuration $C \in \mathcal{C}$ leads to configuration $C^{\prime} \in \mathcal{C}$, written as $T \vdash C \leadsto C^{\prime}$, defined for $s, s^{\prime} \in \Sigma, l, r \in \Sigma^{*}$ and $q, q^{\prime} \in Q$ by

$$
\begin{array}{llll}
T \vdash(q,(l, s, r)) & \sim\left(q^{\prime},\left(l, s^{\prime}, r\right)\right) & \text { if } & \left(q,\left(s, s^{\prime}\right), q^{\prime}\right) \in \delta, \\
T \vdash\left(q,\left(l s^{\prime}, s, r\right)\right) \sim\left(q^{\prime},\left(l, s^{\prime}, s r\right)\right) & \text { if } \quad\left(q, \leftarrow, q^{\prime}\right) & \in \delta, \\
T \vdash(q,(l, s, r)) \leadsto\left(q^{\prime},(l, s, r)\right) & \text { if } \quad\left(q, \downarrow, q^{\prime}\right) & \in \delta, \\
T \vdash\left(q,\left(l, s, s^{\prime} r\right)\right) \leadsto\left(q^{\prime},\left(l s, s^{\prime}, r\right)\right) & \text { if } \quad\left(q, \rightarrow, q^{\prime}\right) & \in \delta .
\end{array}
$$

Definition 4 (Local forward/backward determinism). $A T M T=(Q, \Sigma, \delta$, $\left.b, q_{s}, q_{f}\right)$ is locally forward deterministic iff for any distinct pair of transition rule triples $\left(q_{1}, a_{1}, q_{1}^{\prime}\right),\left(q_{2}, a_{2}, q_{2}^{\prime}\right) \in \delta$, if $q_{1}=q_{2}$ then $a_{1}=\left(s_{1}, s_{1}^{\prime}\right)$ and $a_{2}=\left(s_{2}, s_{2}^{\prime}\right)$, and $s_{1} \neq s_{2}$. A TMT is locally backward deterministic iff for any distinct pair of triples $\left(q_{1}, a_{1}, q_{1}^{\prime}\right),\left(q_{2}, a_{2}, q_{2}^{\prime}\right) \in \delta$, if $q_{1}^{\prime}=q_{2}^{\prime}$ then $a_{1}=\left(s_{1}, s_{1}^{\prime}\right)$ and $a_{2}=\left(s_{2}, s_{2}^{\prime}\right)$, and $s_{1}^{\prime} \neq s_{2}^{\prime}$.

As an example, the pair $(q,(a, b), p)$ and $(q,(a, c), p)$ respects backward determinism (but not forward determinism); the pair $(\mathrm{q},(\mathrm{a}, \mathrm{b}), \mathrm{p})$ and $(\mathrm{r},(\mathrm{c}, \mathrm{b}), \mathrm{p})$ is not backward deterministic; and neither is the pair $(\mathrm{q},(\mathrm{a}, \mathrm{b}), \mathrm{p})$ and $(\mathrm{r}, \rightarrow, \mathrm{p}) 3$.

Definition 5 (Reversible Turing machine). A TM T is reversible iff it is locally forward and backward deterministic.

\footnotetext{
${ }^{2}$ When describing tape contents we shall use the empty string $\varepsilon$ to denote the infinite string of blanks $b^{\omega}$, and shall usually omit it when unambiguous.

${ }^{3}$ When we use typewriter font we usually refer to concrete instances, rather than variables. Thus, in this example $\mathrm{q}$ and $\mathrm{p}$ refers to different concrete states.
} 
The reversible Turing machines (RTMs) are thus a proper subset of the set of all Turing machines, with an easily decidable property. We need the following important lemma. Note that this applies to each computation step.

Lemma 1. If $T$ is a reversible Turing machine, then the induced computation step relation $T \vdash \cdot \leadsto \cdot$ is an injective function on configurations.

\section{Semantics for Turing Machines}

What do Turing machines compute? In other words, what is the codomain and definition of the semantics function $\llbracket \cdot \rrbracket:$ TMs $\rightarrow$ ? for Turing machines? This might seem an odd question seeing as we have just defined how TMs work, but the answer depends on a concrete semantical choice, and has a profound effect on the computational strength of the RTMs. (Note: For the rest of this paper, we shall consider the relationship mainly between deterministic and reversible Turing machines. Thus, all TMs are assumed to be fwd deterministic).

At this point, the expert reader might object that the original results by Landauer 9] and Bennett 4] (cf. Lemmas 4] and 5) show exactly how we can "reversibilize" any computation, and that the RTMs should therefore be able to compute exactly what the TMs in general can compute. Furthermore, Morita and Yamaguchi [13] exhibited a universal reversible Turing machine, so the universality of the RTMs should already be established. As we shall see, however, if one takes reversibility as also including the input/output behaviour of the machines, neither of these claims hold: Reversibilization is not semantics preserving, and the RTMs are not universal in the classical sense.

There are several reasons for considering the extensional behavior of RTMs to itself be subject to reversibility.

- The reversible machines, computation models and programming languages, form a much larger class than just the reversibilized machines of Landauer and Bennett performing reversible simulations of irreversible machines.

- It leads to a richer and more elegant (functional) theory for reversible programs: Program composition becomes function composition, program inversion becomes function inversion, etc., and we are able to use such properties directly and intuitively in the construction of new reversible programs. This is not the case for reversible simulations.

- If we can ad hoc dismiss part of the output configuration, there seems to be little to constrain us from allowing such irreversibilities as part of the computation process as well.

In order to talk about input/output behavior on tapes in a regular fashion, we use the following definition.

Definition 6 (Standard configuration). A tape containing a finite, blankfree string $s \in(\Sigma \backslash\{b\})^{*}$ is said to be given in standard configuration for a TM $\left(Q, \Sigma, \delta, b, q_{s}, q_{f}\right)$ iff the tape head is positioned to the immediate left of $s$ on the tape, i.e. if for some $q \in Q$, the configuration of the $T M$ is $(q,(\varepsilon, b, s))$. 
We shall consider the tape input/output (function) behavior. Here, the semantic function of a Turing machine is defined by its effect on the entire configuration.

Definition 7 (String transformation semantics). The semantics $T T \rrbracket$ of a $T M T=\left(Q, \Sigma, \delta, b, q_{s}, q_{f}\right)$ is given by the relation

$$
\llbracket T \rrbracket=\left\{\left(s, s^{\prime}\right) \in\left((\Sigma \backslash\{b\})^{*} \times(\Sigma \backslash\{b\})^{*}\right) \mid T \vdash\left(q_{s},(\varepsilon, b, s)\right) \sim^{*}\left(q_{f},\left(\varepsilon, b, s^{\prime}\right)\right)\right\} .
$$

Intuitively, a computation is performed as follows. In starting state $q_{s}$, with input $s$ given in standard configuration $\left(q_{s},(\varepsilon, b, s)\right)$, repeatedly apply $\leadsto$, until the machine halts (if it halts) in standard configuration $\left(q_{f},\left(\varepsilon, b, s^{\prime}\right)\right)$. To differentiate between semantics and mechanics, we shall write $T(x)$ to mean the computation of $\llbracket T \rrbracket(x)$ by the specific machine $T$. We say that $T$ computes function $f$ iff $\llbracket T \rrbracket=f$. Thus, the string transformation semantics of a TM $T$ has type

$$
\llbracket T \rrbracket: \Sigma^{*} \rightarrow \Sigma^{*} .
$$

Under this semantics there is a one-to-one correspondence between input/output strings and the configurations that represent them, so the machine input/output behaviour is logically reversible. In contrast to this, the (implicit) semantics used for decision problems (language recognition) gives us programs of type

$$
\llbracket T \rrbracket_{d p}: \Sigma^{*} \rightarrow\{\text { accept, reject }\},
$$

where halting configurations are projected down to a single bit. It is well known that for classical Turing machines it does not matter computability-wise which of these two semantics we choose. (There is a fairly straightforward translation from languages to functions and vice versa.) Anticipating the Landauer embedding of Lemma 4 it is easy to see that under the language recognition semantics then the RTMs are universal: Given a TM $T$ recognizing language $L$, there exists an RTM $T^{\prime}$ that also recognizes $L$. However, under the string transformation semantics the RTMs cannot be universal.

Theorem 1. If $T$ is an RTM, then $\llbracket T \rrbracket$ is injective.

Proof. By induction, using Lemma 1.

It thus makes little sense to talk about what RTMs compute without explicitly specifying the semantics.

\section{Foundations of Reversible Computing}

At this point it becomes necessary to recast the foundational results of reversible computing in terms of the strict semantical interpretation above.

\subsection{Inversion}

If $f$ is a computable injective function, is the inverse function $f^{-1}$ computable? 
Lemma 2 (TM inversion, McCarthy [10]). Given a TM T computing an injective function $\llbracket T \rrbracket$, there exists a $T M M(T)$, such that $\llbracket M(T) \rrbracket=\llbracket T \rrbracket^{-1}$.

It is interesting to note that McCarthy's generate-and-test approach 10 does not actually give the program inverter (computing the transformation $M$ ), but rather an inverse interpreter, cf. 11. However, we can turn an inverse interpreter into a program inverter by specialization [7, so the transformation $M$ is computable.

The generate-and-test method used by McCarthy is sufficient to show the existence of an inverse interpreter, but unsuitable for practical usage as it is very inefficient. For the RTMs there is an appealing alternative.

Lemma 3 (RTM inversion, Bennett [4]). Given an $R T M T=\left(Q, \Sigma, \delta, b, q_{s}\right.$, $\left.q_{f}\right)$, the $R T M T^{-1} \stackrel{\text { def }}{=}\left(Q, \Sigma, i n v(\delta), b, q_{f}, q_{s}\right)$ computes the inverse function of $\llbracket T \rrbracket$, i.e. $\llbracket T^{-1} \rrbracket=\llbracket T \rrbracket^{-1}$, where inv $: \Delta \rightarrow \Delta$ is defined as

$$
\begin{aligned}
& \operatorname{inv}\left(q,\left(s, s^{\prime}\right), q^{\prime}\right)=\left(q^{\prime},\left(s^{\prime}, s\right), q\right) \quad \operatorname{inv}\left(q, \leftarrow, q^{\prime}\right)=\left(q^{\prime}, \rightarrow, q\right) \\
& \operatorname{inv}\left(q, \downarrow, q^{\prime}\right) \quad=\left(q^{\prime}, \downarrow, q\right) \quad \operatorname{inv}\left(q, \rightarrow, q^{\prime}\right)=\left(q^{\prime}, \leftarrow, q\right) \text {. }
\end{aligned}
$$

This remarkably simply transformation is one of the great insights in Bennett's seminal 1973 paper [4 that may superficially seem trivial. Here, we have additionally shown that it is an example of local (peephole) program inversion. Note that the transformation only works as a program inversion under the string transformation semantics, and not under language recognition. In the following, we shall make heavy use of program inversion, so the direct coupling between the mechanical and semantical transformation is significant.

\subsection{Reversibilization}

How can irreversible TMs computing (possibly non-injective) functions be reversibilized, i.e., transformed into RTMs?

Lemma 4 (Landauer embedding [9]). Given a 1-tape $T M T=\left(Q, \Sigma, \delta, b, q_{s}\right.$, $\left.q_{f}\right)$, there is a 2-tape $R T M L(T)$ such that $\llbracket L(T) \rrbracket: \Sigma^{*} \rightarrow \Sigma^{*} \times R^{*}$, and

$$
\llbracket L(T) \rrbracket=\lambda x \cdot(\llbracket T \rrbracket(x), \operatorname{trace}(T, x)),
$$

where trace $(T, x)$ is a complete trace of the specific rules from $\delta$ (enumerated as $R$ ) that are applied during the computation $T(x)$.

The Landauer embedding is named in honor of Rolf Landauer, who suggested the idea of a trace to ensure reversibility 9. It is historically the first example of what we call "reversibilization," the addition of garbage data to the output in order to guarantee reversibility. The Landauer embedding shows that any computable function can be injectivized such that it is computable by a reversible TM.

The size of the garbage data $\operatorname{trace}(T, x)$ is of order of the number of steps in the computation $T(x)$, which makes it in general unsuited for practical programming. The trace is also machine-specific: Given functionally equivalent TMs $T_{1}$ and $T_{2}$, 
i.e., $\llbracket T_{1} \rrbracket=\llbracket T_{2} \rrbracket$, it will almost always be the case that $\llbracket L\left(T_{1}\right) \rrbracket \neq \llbracket L\left(T_{2}\right) \rrbracket$. The addition of the trace also changes the space consumption of the original program.

It is preferable that an injectivization generates extensional garbage data (specific to the function) rather than intensional garbage data (specific to the machine), since we would like to talk about semantics and ignore the mechanics. This is attained in the following Lemma, known colloquially as "Bennett's trick."

Lemma 5 (Bennett's method [4]). Given a 1-tape $T M T=\left(Q, \Sigma, \delta, b, q_{s}, q_{f}\right)$, there exists a 3-tape $R T M B(T)$, s.t.

$$
\llbracket B(T) \rrbracket=\lambda x .(x, \llbracket T \rrbracket(x)) .
$$

While the construction (shown below) is defined for 1-tape machines, it can be extended to Turing machines with an arbitrary number of tapes. It is important to note that neither Landauer embedding nor Bennett's method are semantics preserving as both reversibilizations lead to garbage:

$$
\llbracket L(T) \rrbracket \neq \llbracket T \rrbracket \neq \llbracket B(T) \rrbracket .
$$

\subsection{Reversible Updates}

Bennett's method implements a special case of a reversible update [3, where $D$ (below) is a simple "copying machine", and the second input is initially blank:

Theorem 2. Assume that $\odot:\left(\Sigma^{*} \times \Sigma^{*}\right) \rightarrow \Sigma^{*}$ is a (computable) operator injective in its first argument: If $b \odot a=c \odot a$, then $b=c$. Let $D$ be an RTM computing the injective function $\lambda(a, b) .(a, b \odot a)$, and let $T$ be any TM. Let $L_{1}(T)$ be an RTM that applies $L(T)$ to the first argument $x$ of a pair $(x, y)$ (using an auxiliary tape for the trace.) We havet

$$
\llbracket L_{1}(T)^{-1} \circ D \circ L_{1}(T) \rrbracket=\lambda(x, y) \cdot(x, y \odot \llbracket T \rrbracket(x)) .
$$

Reversible updates models many reversible language constructs [21], and is also useful when designing reversible circuits 16 17. We found this generalization to be of great practical use in the translation from high-level to low-level reversible languages [2], as it directly suggests a translation strategy for reversible updates.

\section{Robustness}

The Turing machines are remarkably computationally robust. Using multiple symbols, tapes, heads etc. has no impact on computability. Above, we have been silently assuming that the same holds true for the RTMs: The Landauer embedding takes $n$-tape machine to $n+1$-tape machines, the Bennett trick takes 1-tape machines to 3 -tape machines, etc.

${ }^{4}$ The mechanical concatenation of two machines $T_{2} \circ T_{1}$ is straightforward, and it is an easy exercise to see that $\llbracket T_{2} \circ T_{1} \rrbracket=\llbracket T_{2} \rrbracket \circ \llbracket T_{1} \rrbracket$. 
Are these assumptions justified? We have seen that a precise characterization of the semantics turned out to have a huge impact on computational expressiveness (limiting us to injective functions.) It would not be unreasonable to expect the RTMs to suffer additional restrictions wrt the parameters of machine space.

First, we consider the question of multiple symbols. Morita et al. [12] showed how to simulate a 1-tape, 32-symbol RTM by a 1-tape 2-symbol RTM. One can generalize this result to an arbitrary number of symbols. Furthermore, we also need to adapt it to work when applying our string transformation semantics such that the encodings can be efficient5.

Lemma 6 ( $m$-symbol RTM to 3-symbol RTM). Given a 1-tape, $m$-symbol $R T M T=\left(Q, \Sigma, \delta, b, q_{s}, q_{f}\right),|\Sigma|=m$, there is a 1-tape, 3-symbol $R T M T^{\prime}=$ $\left(Q^{\prime},\{b, 0,1\}, \delta^{\prime}, b, q_{s}, q_{f}\right)$ s.t. $\llbracket T \rrbracket(x)=y$ iff $\llbracket T^{\prime} \rrbracket(e(x))=e(y)$, where $e:(\Sigma \backslash\{b\})^{*}$ $\rightarrow\{0,1\}^{*}$ is an injective binary encoding of (blank-free) strings, with $b$ encoded by a sequence of blanks.

Thus, the number of symbols in a tape alphabet is not important, and a fixed-size alphabet (with at least 3 distinct symbols) can be used for all computations.

We now turn to the question of multiple tapes.

Lemma 7 (2-tape RTM to 1-tape RTM). Given a 2-tape RTM T, there exists a 1-tape $R T M T^{\prime}$ s.t. $\llbracket T \rrbracket(x, y)=(u, v)$ iff $\llbracket T^{\prime} \rrbracket(\langle x, y\rangle)=\langle u, v\rangle$, where $\langle x, y\rangle=x_{1} y_{1}, x_{2} y_{2}, \ldots$ is the pairwise character sequence (convolution) of strings $x$ and $y$ (with blanks at the end of the shorter string as necessary.)

The main difficulty in proving this is that the original 2-tape machine may allow halting configurations where the tape heads end up displaced an unequal number of cells from their starting positions. Thus "zipping" the tapes into one tape will not necessarily give the convolution of the outputs in standard configuration. This is corrected by realigning the simulated tapes for each rule where the original tape heads move differently.

This result generalizes to an arbitrary number of tapes. Combining these two lemmas yields the following statement of robustness.

Theorem 3 (Robustness of the RTMs). Let $T$ be a $k$-tape, $m$-symbol RTM. Then there exists a 1-tape, 3-symbol RTM $T^{\prime}$ s.t.

$$
\llbracket T \rrbracket\left(x_{1}, \ldots, x_{k}\right)=\left(y_{1}, \ldots, y_{k}\right) \quad \text { iff } \quad \llbracket T^{\prime} \rrbracket\left(e\left(\left\langle x_{1}, \ldots, x_{k}\right\rangle\right)\right)=e\left(\left\langle y_{1}, \ldots y_{k}\right\rangle\right),
$$

where $\langle\cdot\rangle$ is the convolution of tape contents, and $e(\cdot)$ is a binary encoding.

This retroactively justifies the use of the traditional transformational approaches.

\section{Exact Computational Expressiveness of the RTMs}

We have outlined the two classical reversibilizations that turn TMs into RTMs. However, they are not semantics-preserving, and do not tell us anything about

\footnotetext{
${ }^{5}$ A 2-symbol machine can only have unary input strings in standard configuration, as one of the two symbols must be the blank symbol.
} 


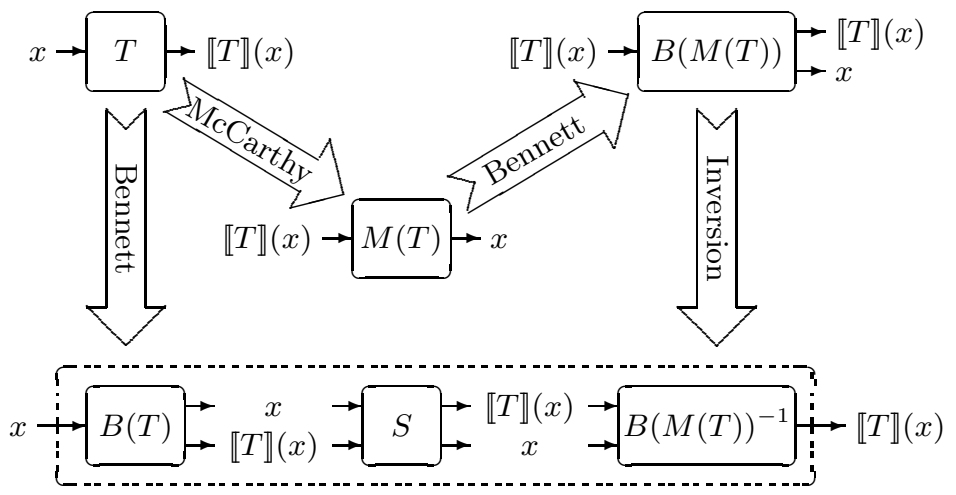

Fig. 1. Generating an RTM computing (injective) $\llbracket T \rrbracket$ from an irreversible TM $T$

the a priori computational expressiveness of RTMs. By Theorem 1 the RTMs compute only injective functions. How many such functions can they compute?

Theorem 4 (Reversibilizing injections, Bennett [4]). Given a 1-tape TM $S_{1}$ s.t. $\llbracket S_{1} \rrbracket$ is injective, and given a 1-tape $T M S_{2}$ s.t. $\llbracket S_{2} \rrbracket=\llbracket S_{1} \rrbracket^{-1}$, there exists a 3-tape RTM T s.t. $\llbracket T \rrbracket=\llbracket S_{1} \rrbracket$.

We can use this to establish the exact computational expressiveness of the RTMs.

Theorem 5 (Expressiveness). The RTMs can compute exactly all injective

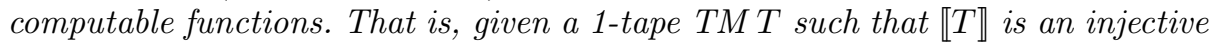
function, there is a 3-tape RTM $T^{\prime}$ such that $\llbracket T \rrbracket=\llbracket T^{\prime} \rrbracket$.

This theorem then follows from the existence of a TM inverter (Lemma 2) and Theorem 4. We make use of the construction used by Bennett to prove Theorem 4 , but now operating purely at the semantics level, making direct use of the transformations, and without assuming that an inverse for $T$ is given a priori.

Proof. We construct and concatenate three RTMs (see Fig. 1 for a graphical representation.) First, construct $B(T)$ by applying Lemma 5 directly to $T$ :

$$
\llbracket B(T)) \rrbracket=\lambda x .(x, \llbracket T \rrbracket(x)), \quad B(T) \in \mathrm{RTMs}
$$

Second, construct the machine $B(M(T))^{-1}$ by successively applying the transformations of Lemmas 2, 5 and 3 to $T$ :

$$
\llbracket B(M(T))^{-1} \rrbracket=\left(\lambda y \cdot\left(y, \llbracket T \rrbracket^{-1}(y)\right)\right)^{-1}, \quad B(M(T))^{-1} \in \mathrm{RTMs}
$$

Third, we can construct an RTM $S$, s.t. $\llbracket S \rrbracket=\lambda(a, b) .(b, a)$, that is, a machine to exchange the contents of two tapes (in standard configuration). To see that $\llbracket B(M(T))^{-1} \circ S \circ B(T) \rrbracket=\llbracket T \rrbracket$, we apply the machine to an input, $x$ : 


$$
\begin{aligned}
\llbracket B(M(T))^{-1} \circ S \circ B(T) \rrbracket(x) & =\llbracket B(M(T))^{-1} \circ \llbracket S \rrbracket \rrbracket(x, \llbracket T \rrbracket(x)) \\
& \left.=\llbracket B(M(T))^{-1} \rrbracket(\llbracket T \rrbracket(x), x)\right) \\
& =\left(\lambda y \cdot\left(y, \llbracket T \rrbracket^{-1}(y)\right)\right)^{-1}(\llbracket T \rrbracket(x), x) \\
& =\left(\lambda y \cdot\left(y, \llbracket T \rrbracket^{-1}(y)\right)\right)^{-1}\left(\llbracket T \rrbracket(x), \llbracket T \rrbracket^{-1}(\llbracket T \rrbracket(x))\right) \\
& =\llbracket T \rrbracket(x) .
\end{aligned}
$$

Thus, the RTMs can compute exactly all the injective computable functions. This suggests that the RTMs have the maximal computational expressiveness we could hope for in any (effective) reversible computing model.

\section{Universality}

Having characterized the computational expressiveness of the RTMs, an obvious next question is that of computation universality. A universal machine is a machine that can simulate the functional behaviour of any other machine. For the classical, irreversible Turing machines, we have the following definition.

Definition 8 (Classical universality). A TMU is classically universal iff for all TMs $T$, all inputs $x \in \Sigma^{*}$, and Gödel number $\ulcorner T\urcorner \in \Sigma^{*}$ representing $T$ :

$$
\llbracket U \rrbracket(\ulcorner T\urcorner, x)=\llbracket T \rrbracket(x) .
$$

The actual Gödel numbering $\ulcorner\urcorner:$. TMs $\rightarrow \Sigma^{*}$ for a given universal machine is not important, but we do require that it is computable and injective (up to renaming of symbols and states).

Because $\llbracket U \rrbracket$ in this definition is a non-injective function, it is clear that no classically universal RTM exists! Bennett [4] suggests that if $U$ is a (classically) universal machine, $B(U)$ is a machine for reversibly simulating any irreversible machine. However, $B(U)$ is not itself universal, $\llbracket B(U) \rrbracket \neq \llbracket U \rrbracket$, and furthermore we should not use reversible simulation of irreversible machines as a benchmark.

The appropriate question to ask is whether the RTMs are classically universal for just their own class, i.e. where the interpreted machine $T$ is restricted to being an RTM. The answer is, again, no: Different programs may compute the same function, so there exists RTMs $T_{1} \neq T_{2}$ such that $\llbracket T_{1} \rrbracket(x)=\llbracket T_{2} \rrbracket(x)$, so $\llbracket U \rrbracket$ is inherently non-injective, and therefore not computable by any RTM.

Classical universality is thus unsuitable if we want to capture a similar notion wrt RTMs. We propose that a universal machine should be allowed to remember which machine it simulates.

Definition 9 (Universality). A TM $U_{T M}$ is universal iff for all TMs $T$ and all inputs $x \in \Sigma^{*}$,

$$
\llbracket U_{T M} \rrbracket(\ulcorner T\urcorner, x)=(\ulcorner T\urcorner, \llbracket T \rrbracket(x)) .
$$


This is equivalent to the original definition of classical universality 6 . Importantly, it now suggests a concept of universality that can apply to RTMs.

Definition 10 (RTM-universality). An RTM $U_{R T M}$ is RTM-universal iff for all RTMs $T$ and all inputs $x \in \Sigma^{*}$,

$$
\llbracket U_{R T M} \rrbracket(\ulcorner T\urcorner, x)=(\ulcorner T\urcorner, \llbracket T \rrbracket(x)) .
$$

Now, is there an RTM-universal reversible Turing machine, a URTM?

Theorem 6 (URTM existence). There exists an RTM-universal RTM $U_{R}$.

Proof. We show that an RTM $U_{R}$ exits, such that for all RTMs $T, \llbracket U_{R} \rrbracket(\ulcorner T\urcorner, x)=$ $(\ulcorner T\urcorner, \llbracket T \rrbracket(x))$. Clearly, $\llbracket U_{R} \rrbracket$ is computable, since $T$ is a TM (so $\llbracket \rrbracket$ is computable), and $\ulcorner T\urcorner$ is given as input. We show that $\llbracket U_{R} \rrbracket$ is injective: Assuming $\left(\left\ulcorner T_{1}\right\urcorner, x_{1}\right) \neq\left(\left\ulcorner T_{2}\right\urcorner, x_{2}\right)$ we show that $\left(\left\ulcorner T_{1}\right\urcorner, \llbracket T_{1} \rrbracket\left(x_{1}\right)\right) \neq\left(\left\ulcorner T_{2}\right\urcorner, \llbracket T_{2} \rrbracket\left(x_{2}\right)\right)$. Either $\left\ulcorner T_{1}\right\urcorner \neq\left\ulcorner T_{2}\right\urcorner$ or $x_{1} \neq x_{2}$ or both. Because the program text is passed through to the output, the first and third cases are trivial. Assuming that $x_{1} \neq x_{2}$ and $\left\ulcorner T_{1}\right\urcorner=\left\ulcorner T_{2}\right\urcorner$, we have that $\llbracket T_{1} \rrbracket=\llbracket T_{2} \rrbracket$, i.e. $T_{1}$ and $T_{2}$ are the same machine, and so compute the same function. Because they are RTMs this function is injective (by Theorem 1), so $x_{1} \neq x_{2}$ implies that $\llbracket T_{1} \rrbracket\left(x_{1}\right) \neq \llbracket T_{2} \rrbracket\left(x_{2}\right)$. Therefore, $\llbracket U_{R} \rrbracket$ is injective, and by Theorem 5 computable by some RTM $U_{R}$.

We remark that this works out very nicely: RTM-universality is now simply universality restricted to interpreting the RTMs, and while general universality is non-injective, RTM-universality becomes exactly injective by virtue of the semantics of RTMs. Also, by interpreting just the RTMs, we remove the redundancy (and reliance on reversibilization) inherent in the alternatives.

Given an irreversible TM computing the function of RTM-universality, Theorem 5 provides us with a possible construction for an RTM-universal RTM. However, we do not actually directly have such machines in the literature, and in any case the construction uses the very inefficient generate-and-test inverter by McCarthy. We can do better.

Lemma 8. There exists an RTM pinv, such that pinv is a program inverter for RTM programs,

$$
\llbracket p i n v \rrbracket(\ulcorner T\urcorner)=\left\ulcorner T^{-1}\right\urcorner .
$$

This states that the RTMs are expressive enough to perform the program inversion of Lemma 3. For practical Gödelizations this will only take linear time.

Theorem 7 (UTM to URTM). Given a classically universal TMU s.t. $\llbracket U \rrbracket(\ulcorner T\urcorner, x)=\llbracket T \rrbracket(x)$, the $R T M U_{R}$ defined as follows is RTM-universal.

$$
U_{R}=\operatorname{pinv}_{1} \circ(B(U))^{-1} \circ S_{23} \circ \operatorname{pinv}_{1} \circ B(U),
$$

where pinv $_{1}$ is an RTM that applies RTM program inversion on its first argument, $\llbracket p i n v_{1} \rrbracket(p, x, y)=(\llbracket p i n v \rrbracket p, x, y)$, and $S_{23}$ is an RTM that swaps its second and third arguments, $\llbracket S_{23} \rrbracket=\lambda(x, y, z) \cdot(x, z, y)$.

${ }^{6}$ Given $U_{T M}$ universal by Definition 9 snd $\circ U_{T M}$ is classically universal, where $s n d$ is a TM s.t. $\llbracket s n d \rrbracket=\lambda(x, y) . y$. The converse is analogous. 


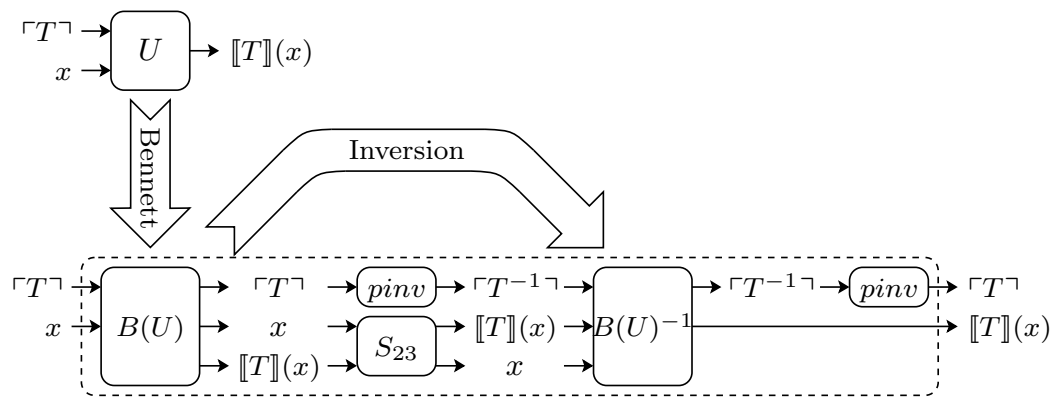

Fig. 2. Constructing an RTM-universal RTM $U_{R}$ from a classically universal TM $U$

Proof. We must show that $\llbracket U_{R} \rrbracket(\ulcorner T\urcorner, x)=(\ulcorner T\urcorner, \llbracket T \rrbracket(x))$ for any RTM $T$. To show this, we apply $U_{R}$ to an input $(\ulcorner T\urcorner, x)$. Fig. 2 shows a graphical representation of the proof.

$$
\begin{aligned}
& \llbracket U_{R} \rrbracket(\ulcorner T\urcorner, x)=\llbracket \operatorname{pinv}_{1} \circ(B(U))^{-1} \circ S_{23} \circ \text { pinv }_{1} \circ B(U) \rrbracket(\ulcorner T\urcorner, x) \\
& =\llbracket \text { pinv }_{1} \circ(B(U))^{-1} \circ S_{23} \circ \text { pinv }_{1} \rrbracket(\ulcorner T\urcorner, x, \llbracket T \rrbracket(x)) \\
& =\llbracket \text { pinv }_{1} \circ(B(U))^{-1} \circ S_{23} \rrbracket\left(\left\ulcorner T^{-1}\right\urcorner, x, \llbracket T \rrbracket(x)\right) \\
& =\llbracket \text { pinv }_{1} \circ(B(U))^{-1} \rrbracket\left(\left\ulcorner T^{-1}\right\urcorner, \llbracket T \rrbracket(x), x\right) \\
& =\llbracket \text { pinv }_{1} \rrbracket\left(\left\ulcorner T^{-1}\right\urcorner, \llbracket T \rrbracket(x)\right) \\
& =(\ulcorner T\urcorner, \llbracket T \rrbracket(x)) \text {. }
\end{aligned}
$$

Note that this implies that RTMs can simulate themselves exactly as timeefficiently as the TMs can simulate themselves, but the space usage of the constructed machine will, by using Bennett's method, be excessive. However, there is nothing that forces us to start with an irreversible (universal) machine, when constructing an RTM-universal RTM, nor are reversibilizations necessarily required (as will be seen below).

A first principles approach to an RTM-universal reversible Turing machine, which does not rely on reversibilization, remains for future work.

\section{8 r-Turing Completeness}

With a theory of the computational expressiveness and universality of the RTMs at hand, we shall lift the discussion to computation models in general. What, then, do reversible programs compute, and what can they compute?

Our fundamental assumption is that the RTMs (with the given semantics) are a good and exhaustive model for reversible computing. Thus, for every program $\mathrm{p}$ in a reversible programming language $R$, we assume there to be an RTM $T_{\mathrm{p}}$, s.t. $\llbracket \mathrm{p} \rrbracket_{R}=\llbracket T_{\mathrm{p}} \rrbracket$. Thus, because the RTMs are restricted to computing injective functions, reversible programs too compute injective functions only. On the other hand, we have seen that the RTMs are maximally expressive wrt these functions, 
and support a natural notion of universality. For this reason we propose the following standard of computability for reversible computing.

Definition 11 (r-Turing completeness). A (reversible) programming language $R$ is called r-Turing complete iff for all RTMs T computing function $\llbracket T \rrbracket$, there exists a program $\mathrm{p} \in R$, such that $\llbracket \mathrm{p} \rrbracket_{R}=\llbracket T \rrbracket$.

Note that we are here quite literal about the semantics: Given an RTM $T$, it will not suffice to compute a Landauer embedded version of $\llbracket T \rrbracket$, or apply Bennett's trick, or, indeed any injectivization of $\llbracket T \rrbracket$. Program p must compute $\llbracket T \rrbracket$, exactly. Only if this is respected can we truly say that a reversible computation model can compute all the injective, computable functions, i.e. is as computationally expressive as we can expect reversible computation models to be.

Demonstrating r-Turing Completeness. A common approach to proving that a language, or computational model, is Turing-complete, is to demonstrate that a classically universal TM (a TM interpreter) can be implemented, and specialized to any TM T. However, that is for classically universal machines and (in general) irreversible languages, which compute non-injective functions. What about our notion of RTM-universality (Definition 10) and reversible languages?

Assume that $u \in R$ (where $R$ is a reversible programming language) is an $R$ program computing an RTM-universal interpreter $\llbracket u \rrbracket_{R}(\ulcorner T\urcorner, x)=(\ulcorner T\urcorner, \llbracket T \rrbracket(x))$. Assume also that $R$ is expressive enough to guarantee the existence of programs $w_{T} \in R$ s.t. $\llbracket w_{T} \rrbracket_{R}=\lambda x .(\ulcorner T\urcorner, x)$, (whose sole purpose is to hardcode $\ulcorner T\urcorner$ as an input for $u$ ) and its inverse $w_{T}^{-1} \in R, \llbracket w_{T}^{-1} \rrbracket_{R}=\llbracket w_{T} \rrbracket_{R}^{-1}$, for any RTM T. Note that $\llbracket w_{T} \rrbracket_{R}$ is injective, so we do not violate our rule of $R$ computing injective functions by assuming $w_{T}$ and its inverse. Now $\llbracket u \circ w_{T} \rrbracket_{R}=\lambda x .(\ulcorner T\urcorner, \llbracket T \rrbracket(x)) \neq$ $\llbracket T \rrbracket$, because it leaves the representation $\ulcorner T\urcorner$ as part of the output. To complete the specialization, we need to apply $w_{T}^{-1}$ as well. Thus, $\llbracket w_{T}^{-1} \circ u \circ w_{T} \rrbracket_{R}=\llbracket T \rrbracket$.

Therefore, completely analogous to the classical case, we may demonstrate $\mathrm{r}-$ Turing completeness of a reversible computation model by implementing RTMuniversality (essentially, an RTM interpreter), keeping in mind that we must respect the semantics exactly (by clean simulation that doesn't leave garbage).

The authors have done exactly that to demonstrate r-Turing completeness of the imperative, high level, reversible language Janus, and for reversible flowchart languages in general, cf. 2122] (where the r-Turing completeness concept was informally proposed.) In these cases, we were able to exploit the reversibility of the interpreted machines directly, and did not have to rely on reversibilization of any kind, which eased the implementation greatly. Furthermore, the RTMinterpreters are complexity-wise robust, in that they preserve the space and time complexities of the simulated machines, which no reversibilization is liable to do.

\section{Related Work}

Morita et al. have studied reversible Turing machines 1312 and other reversible computation models, including cellular automata [1], with a different approach 
to semantics (and thus different results wrt computational strength) than the present paper. Most relevant here is the universal RTM proposed in [13. With our strict semantics viewpoint, the construction therein does not directly demonstrate neither RTM-universality nor classical universality, but rather a sort of "traced" universality: Given a program for a cyclic tag system (a Turing complete formalism) and an input, the halting configuration encompasses both the program and output, but also the entire string produced by the tag system along the way. We believe that this machine could possibly be transformed fairly straightforwardly into a machine computing a function analogous to $\llbracket B(U) \rrbracket$. However, it is not clear that cyclic tag systems should have a notion of reversibility, so the construction in Fig. 2 is therefore not immediately applicable.

\section{Conclusion}

The study of reversible computation models complements that of deterministic and non-deterministic computation models. We have given a foundational treatment of a computability theory for reversible computing using a strict semanticsbased approach (where input/output behaviour must also be reversible), taking reversible Turing machines as the underlying computational model. By formulating the classical transformational approaches to reversible computation in terms of this semantics, we hope to have clarified the distinction between reversibility and reversibilization, which may previously have been unclear.

We found that starting directly with reversibility leads to a clearer, cleaner, and more useful functional theory for RTMs. Natural (mechanical) program transformations such as composition and inversion now correspond directly to the (semantical) function transformations. This carries over to other computation models as well.

We showed that the RTMs compute exactly all injective, computable functions, and are thus not classically universal. We also showed that they are expressive enough to be universal for their own class, with the concept of RTMuniversality. We introduced the concept of $r$-Turing completeness as the measure of the computational expressiveness in reversible computing. As a consequence, a definitive practical criterion for deciding the computation universality of a reversible programming computation model is now in place: Implement an RTMinterpreter, in the sense of an RTM-universal machine.

Acknowledgements. The authors wish to thank Michael Kirkedal Thomsen for help with the figures and Tetsuo Yokoyama for discussions on RTM-computability.

\section{References}

1. Abramov, S., Glück, R.: Principles of inverse computation and the universal resolving algorithm. In: Mogensen, T.æ., Schmidt, D.A., Sudborough, I.H. (eds.) The Essence of Computation. LNCS, vol. 2566, pp. 269-295. Springer, Heidelberg (2002) 
2. Axelsen, H.B.: Clean translation of an imperative reversible programming language. In: Knoop, J. (ed.) CC 2011. LNCS, vol. 6601, pp. 144-163. Springer, Heidelberg (2011)

3. Axelsen, H.B., Glück, R., Yokoyama, T.: Reversible machine code and its abstract processor architecture. In: Diekert, V., Volkov, M.V., Voronkov, A. (eds.) CSR 2007. LNCS, vol. 4649, pp. 56-69. Springer, Heidelberg (2007)

4. Bennett, C.H.: Logical reversibility of computation. IBM Journal of Research and Development 17, 525-532 (1973)

5. Feynman, R.: Quantum mechanical computers. Optics News 11, 11-20 (1985)

6. Foster, J.N., Greenwald, M.B., Moore, J.T., Pierce, B.C., Schmitt, A.: Combinators for bi-directional tree transformations: A linguistic approach to the view update problem. ACM Trans. Prog. Lang. Syst. 29(3), article 17 (2007)

7. Glück, R., Sørensen, M.: A roadmap to metacomputation by supercompilation. In: Danvy, O., Thiemann, P., Glück, R. (eds.) Partial Evaluation. LNCS, vol. 1110, pp. 137-160. Springer, Heidelberg (1996)

8. Jones, N.D.: Computability and Complexity: From a Programming Language Perspective. In: Foundations of Computing. MIT Press, Cambridge (1997)

9. Landauer, R.: Irreversibility and heat generation in the computing process. IBM Journal of Research and Development 5(3), 183-191 (1961)

10. McCarthy, J.: The inversion of functions defined by Turing machines. In: Automata Studies, pp. 177-181. Princeton University Press, Princeton (1956)

11. Morita, K.: Reversible computing and cellular automata - A survey. Theoretical Computer Science 395(1), 101-131 (2008)

12. Morita, K., Shirasaki, A., Gono, Y.: A 1-tape 2-symbol reversible Turing machine. Trans. IEICE, E 72(3), 223-228 (1989)

13. Morita, K., Yamaguchi, Y.: A universal reversible turing machine. In: DurandLose, J., Margenstern, M. (eds.) MCU 2007. LNCS, vol. 4664, pp. 90-98. Springer, Heidelberg (2007)

14. Mu, S.-C., Hu, Z., Takeichi, M.: An algebraic approach to bi-directional updating. In: Chin, W.-N. (ed.) APLAS 2004. LNCS, vol. 3302, pp. 2-20. Springer, Heidelberg (2004)

15. Schellekens, M.: MOQA; unlocking the potential of compositional static averagecase analysis. Journal of Logic and Algebraic Programming 79(1), 61-83 (2010)

16. Thomsen, M.K., Axelsen, H.B.: Parallelization of reversible ripple-carry adders. Parallel Processing Letters 19(2), 205-222 (2009)

17. Thomsen, M.K., Glück, R., Axelsen, H.B.: Reversible arithmetic logic unit for quantum arithmetic. Journal of Physics A: Mathematics and Theoretical 42(38), $2002(2010)$

18. Toffoli, T.: Reversible computing. In: de Bakker, J.W., van Leeuwen, J. (eds.) ICALP 1980. LNCS, vol. 85, pp. 632-644. Springer, Heidelberg (1980)

19. van de Snepscheut, J.L.A.: What computing is all about. Springer, Heidelberg (1993)

20. Van Rentergem, Y., De Vos, A.: Optimal design of a reversible full adder. International Journal of Unconventional Computing 1(4), 339-355 (2005)

21. Yokoyama, T., Axelsen, H.B., Glück, R.: Principles of a reversible programming language. In: Proceedings of Computing Frontiers, pp. 43-54. ACM Press, New York (2008)

22. Yokoyama, T., Axelsen, H.B., Glück, R.: Reversible flowchart languages and the structured reversible program theorem. In: Aceto, L., Damgård, I., Goldberg, L.A., Halldórsson, M.M., Ingólfsdóttir, A., Walukiewicz, I. (eds.) ICALP 2008, Part II. LNCS, vol. 5126, pp. 258-270. Springer, Heidelberg (2008) 